\title{
Spin-Magnetic Moment of Dirac Electron, and Role of Zitterbewegung
}

\author{
Shigeru Sasabe \\ Department of Science \& Technology, Tokyo Metropolitan University, Tokyo, Japan \\ Email: s-sasabe@tmu.ac.jp
}

Received 20 December 2013; revised 18 January 2014; accepted 15 February 2014

Copyright (C) 2014 by author and Scientific Research Publishing Inc.

This work is licensed under the Creative Commons Attribution International License (CC BY).

http://creativecommons.org/licenses/by/4.0/

(c) (i) Open Access

\begin{abstract}
The spin-magnetic moment of the electron is revisited. In the form of the relativistic quantum mechanics, we calculate the magnetic moment of Dirac electron with no orbital angular-momentum. It is inferred that obtained magnetic moment may be the spin-magnetic moment, because it is never due to orbital motion. A transition current flowing from a positive energy state to a negative energy state in Dirac Sea is found. Application to the band structure of semiconductor is suggested.
\end{abstract}

\section{Keywords}

Spin-Magnetic Moment, Zitterbewegung, G-Factor, Dirac Electron, Band Structure, Semiconductor

\section{Introduction}

The spin and the spin-magnetic moment are basic and the most important concepts in the spintronics [1] that is new research field in considerable expansion. In the previous work [2], we found that the spin-magnetic moment seems to be caused from well-known definitional equation of magnetic moment. Such a case never happen in the non-relativistic quantum mechanics. In the relativistic quantum mechanics, however, the electron has another degree of freedom called Zitterbewegung [3]-[5] that is trembling motion of relativistic electron. Some physical connection between the spin-magnetic moment and Zitterbewegung was implied in the previous work [2].

In this paper, we investigate a question about the origin of the spin-magnetic moment of the electron and roles of Zitterbewegung relating to it. The use of Heisenberg picture will make hidden roles of Zitterbewegung more clear than previous work. On the other hand, Zitterbewegung in solid state physics [6]-[8] has been a subject of great interest in recent years, since observable Zitterbewegung-like dynamics of band electron was predicted [9] [10] for electron moving in narrow-gap semiconductors [11], graphene sheets [12], carbon nanotube [13], and super conductor [14]. Our research in this paper is therefore worthwhile on both sides of science and technology. As a result, we obtain 


$$
\left\langle\mu_{z, 11}\right\rangle=\frac{e \hbar c}{2 E_{p}}
$$

more easily than the previous work [2], where $\left\langle\mu_{z, 11}\right\rangle$ denotes expectation value of $z$-component of spin-magnetic moment of a free Dirac electron in positive energy state.

\section{Relation between Spin and Spin-Magnetic Moment}

It is well known that the relativistic electron put in the external magnetic field gives interaction energy with the magnetic field $\boldsymbol{H}^{\text {ext }}$. This term [15] [16]

$$
-\frac{e \hbar}{2 m c} \sigma^{\prime} \boldsymbol{H}^{e x t}
$$

was understood as the interaction energy $-\boldsymbol{\mu} \cdot \boldsymbol{H}^{\text {ext }}$ between an external magnetic field $\boldsymbol{H}^{\text {ext }}$ and the magnetic moment $\boldsymbol{\mu}$. Then, physicists concluded that

$$
\boldsymbol{\mu}=\frac{e \hbar}{2 m c} \sigma^{\prime}
$$

must be the spin-magnetic moment of the electron in comparison with Equation (2). However, Equation (3) provided merely the relation of the spin-magnetic moment $\mu$ and the spin operator $\hbar \sigma^{\prime} / 2$ by the analogy with classical electrodynamics. We do not still know how the spin-magnetic moment is generated, and what the spin-magnetic moment is. In order to clarify the origin of the spin-magnetic moment, we must deduce it without the external magnetic field which always leads to the interaction energy of the form $-\boldsymbol{\mu} \cdot \boldsymbol{H}$. Generally, the magnetic moment for a charged particle moving with the velocity $\boldsymbol{v}$ and the charge $e$ is defined as [17] [18]

$$
\boldsymbol{\mu}=\frac{e}{2 c} \boldsymbol{r} \times \boldsymbol{v}
$$

in the classical electrodynamics, where $c$ is speed of light. In the non-relativistic quantum theory, the above equation can be expressed as

$$
\boldsymbol{\mu}=\frac{e}{2 m c} \boldsymbol{L}
$$

by using $\boldsymbol{v}=\boldsymbol{p} / m$ and $\boldsymbol{L}=\boldsymbol{r} \times \boldsymbol{p}$.

In relativistic quantum theory, however, we should mind that we can not use Equation (5) for Dirac electron, because the velocity $\boldsymbol{v}$ and the momentum $\boldsymbol{p}$ are independent variables to each other (i.e. $\boldsymbol{v} \neq \boldsymbol{p} / \mathrm{m}$ ) in this case [2].

\section{Zitterbewegung}

As to Zitterbewegung, we briefly show all equations that are needed later. The velocity $v$ of Dirac electron is given [19] by Heisenberg equation,

$$
\boldsymbol{v} \equiv \dot{\boldsymbol{r}}=\frac{1}{i \hbar}[\boldsymbol{r}, H]=c \boldsymbol{\alpha},
$$

where $\boldsymbol{\alpha}=\left(\alpha_{x}, \alpha_{y}, \alpha_{z}\right)$ are the Dirac matrices in Dirac Hamiltonian

$$
H=c \boldsymbol{\alpha} \boldsymbol{p}+\beta m c^{2} .
$$

The $4 \times 4$ matrices $\alpha$ and $\beta$ are defined as

$$
\alpha=\left[\begin{array}{ll}
0 & \sigma \\
\sigma & 0
\end{array}\right], \quad \beta=\left[\begin{array}{cc}
1 & 0 \\
0 & -1
\end{array}\right],
$$

where $\sigma=\left(\sigma_{x}, \sigma_{y}, \sigma_{z}\right)$ are the Pauli matrices. These matrices satisfy the following relations:

$$
\alpha_{i}^{2}=\beta^{2}=1 \quad(i=x, y, z)
$$




$$
\left\{\alpha_{i}, \alpha_{j}\right\}=2 \delta_{i, j}, \quad\left\{\alpha_{i}, \beta\right\}=0
$$

In order to clarify the role of Zitterbewegung, we use Heisenberg picture hereafter to calculate the time evolution of any operator. We first investigate the behavior of matrix $\alpha_{i}(t)$ as an operator $v_{i}(t) / c$. Making use of Equation (10), we easily find [4]

$$
i \hbar \dot{\alpha}_{i}(t)=2 \alpha_{i}(t) H-2 c p_{i} \quad(i=x, y, z),
$$

where $\alpha_{i}(0)$ is the original matrix $\alpha_{i}$ of Equation (7) in schrödinger picture. Differentiation of both sides of Equation (11) by $t$ gives

$$
\ddot{\alpha}_{i}(t)=\frac{2}{i \hbar} \dot{\alpha}_{i}(t) H .
$$

The solution of the above differential equation is

$$
\dot{\alpha}_{i}(t)=\dot{\alpha}_{i}(0) \exp \left(\frac{2 H t}{i \hbar}\right) .
$$

We substitute Equation (13) into Equation (11) to obtain

$$
\alpha_{i}(t)=\frac{i \hbar}{2} \dot{\alpha}_{i}(0) \exp \left(\frac{2 H t}{i \hbar}\right) H^{-1}+c p_{i} H^{-1} .
$$

Taking $t=0$, we have the above relation in another form.

$$
\dot{\alpha}_{i}(0)=\frac{2}{i \hbar}\left(\alpha_{i}(0) H-c p_{i}\right) .
$$

We finally obtain

$$
\alpha_{i}(t)=\left(\alpha_{i}(0)-c p_{i} H^{-1}\right) \exp \left(\frac{2 H t}{i \hbar}\right)+c p_{i} H^{-1}
$$

from Equations (15) and (14).

\section{Solutions of Dirac Equation}

For reader's convenience, we summarize all equations in the following; they are necessary for our calculation. The Dirac equation

$$
\left(c \boldsymbol{\alpha} \boldsymbol{p}+\beta m c^{2}\right) \psi=E \psi
$$

has four eigen-solutions. We name these solutions $\left|E_{s}(p)\right\rangle(s=1,2,3,4)$ defined as

$$
\left|E_{s}(\boldsymbol{p})\right\rangle= \begin{cases}\left|+E_{p}, \uparrow\right\rangle & (s=1) \\ \left|+E_{p}, \downarrow\right\rangle & (s=2) \\ \left|-E_{p}, \uparrow\right\rangle & (s=3) \\ \left|-E_{p}, \downarrow\right\rangle & (s=4)\end{cases}
$$

where $E_{p}$ is the energy of a free Dirac electron with momentum $\boldsymbol{p}$,

$$
E_{p}=\sqrt{p^{2} c^{2}+m^{2} c^{4}}
$$

Two arrows $\uparrow$ and $\downarrow$ denote 'Up Spin' and 'Down Spin' respectively. The explicit forms of eigensolutions in Heisenberg picture are given by

$$
\psi_{s}(\boldsymbol{r}) \equiv\left\langle\boldsymbol{r} \mid E_{s}(\boldsymbol{p})\right\rangle=\frac{1}{\sqrt{V}} u_{s}(\boldsymbol{p}) \exp \left(\frac{i \boldsymbol{p r}}{\hbar}\right),
$$

where $V$ is normalization volume, and $u_{s}(p)$ are expressed as follows [16]; 


$$
\begin{aligned}
& u_{1}(\boldsymbol{p})=\frac{1}{N}\left[\begin{array}{c}
\chi_{\uparrow} \\
\frac{c \boldsymbol{\sigma} \boldsymbol{p}}{m c^{2}+E_{p}} \chi_{\uparrow}
\end{array}\right], \\
& u_{2}(\boldsymbol{p})=\frac{1}{N}\left[\begin{array}{c}
\chi_{\downarrow} \\
\frac{c \boldsymbol{\sigma} \boldsymbol{p}}{m c^{2}+E_{p}} \chi_{\downarrow}
\end{array}\right], \\
& u_{3}(\boldsymbol{p})=\frac{1}{N}\left[\begin{array}{c}
\frac{-c \boldsymbol{\sigma} \boldsymbol{p}}{m c^{2}+E_{p}} \chi_{\uparrow} \\
\chi_{\uparrow}
\end{array}\right], \\
& u_{4}(\boldsymbol{p})=\frac{1}{N}\left[\begin{array}{c}
\frac{-c \boldsymbol{\sigma} \boldsymbol{p}}{m c^{2}+E_{p}} \chi_{\downarrow} \\
\chi_{\downarrow}
\end{array}\right] .
\end{aligned}
$$

with normalization factor

$$
N=\sqrt{\frac{2 E_{p}}{m c^{2}+E_{p}}} .
$$

and eigen states of "Up Spin” and "Down Spin",

$$
\chi_{\uparrow}=\left[\begin{array}{l}
1 \\
0
\end{array}\right], \quad \chi_{\downarrow}=\left[\begin{array}{l}
0 \\
1
\end{array}\right],
$$

respectively. The momentum $\boldsymbol{p}$ takes discrete values in normalization volume $V=L^{3}$ : That is $p_{i}=2 \pi n_{i} \hbar / L$ $\left(n_{i}= \pm 1, \pm 2, \cdots\right)$ for $i=x, y, z$. The functions $u_{s}(\boldsymbol{p})$ are orthonormalized:

$$
u_{r}^{\dagger}(\boldsymbol{p}) u_{s}(\boldsymbol{p})=\delta_{r s},
$$

as well as

$$
\left\langle E_{r}(\boldsymbol{p}) \mid E_{s}(\boldsymbol{q})\right\rangle=\delta_{r s} \delta_{\boldsymbol{p q}} .
$$

Next relations are especially important in a frame $\boldsymbol{p}=(0,0, p)$ :

$$
\begin{gathered}
\alpha_{x} u_{1}(p)=u_{4}(p) \\
\alpha_{y} u_{1}(p)=i u_{4}(p) .
\end{gathered}
$$

Because each component of $\alpha$ satisfies $\alpha_{i}^{2}=1, \alpha_{i}$ takes the eigenvalues +1 and -1 . This means the velocity $v_{i}$ also has two eigenvalues $v_{i}= \pm c$, that is the speed of light. However, states $\left|E_{r}\right\rangle$ are not eigen states of $v_{i}$. An explicit form of $\alpha_{i}(t)$ in the next section is applicable to the states $\left|E_{r}\right\rangle$.

\section{Expectation Value of Zitterbewegung}

In actual calculation, we will take z-axis along the momentum of the electron: $\boldsymbol{p}=(0,0, p)$. This procedure is necessary in order to not only simplify our calculation but also exclude the z-component of angular momentum caused by orbital motion of the electron.

The velocity operator $\boldsymbol{v}=\boldsymbol{c} \boldsymbol{\alpha}$ is divided into two parts.

$$
v_{i}(t)=v_{i}(t)^{(Z i t)}+v_{i}(t)^{(\text {unif })},
$$

where

$$
v_{i}(t)^{(Z i t)}=\left(c \alpha_{i}(0)-c^{2} p_{i} H^{-1}\right) \exp \left(\frac{2 H t}{i \hbar}\right)
$$

and 


$$
v_{i}(t)^{(\text {unif })}=c^{2} p_{i} H^{-1},
$$

$(i=x, y, z)$ from Equation (16). The velocity $v_{i}^{(Z i t)}$ is Zitterbewegung part which includes oscillation factor, and $v_{i}^{(\text {unif })}$ corresponds to uniform velocity. The coordinate operator $\boldsymbol{r}=(x, y, z)$ is also easily calculated from corresponding part of the above equations.

$$
\begin{gathered}
r_{i}(t)=r_{i}(t)^{(Z i t)}+r_{i}(t)^{(\text {unif })}, \\
r_{i}(t)^{(Z i t)}=\frac{i \hbar}{2}\left(c \alpha_{i}(0)-c^{2} p_{i} H^{-1}\right) H^{-1} \exp \left(\frac{2 H t}{i \hbar}\right)+R_{i} \\
r_{i}(t)^{(\text {unif })}=c^{2} p_{i} H^{-1} t+r_{0, i}
\end{gathered}
$$

where $R_{i}$ is an integration constant, and $r_{0, i}$ agrees to an initial point of the electron in classical sense. Remembering that $\alpha(0)$ is equal to the original $\alpha$ of Equation (7), we easily calculate the expectation value of $c \alpha_{z}$ for $\left|E_{1}\right\rangle$ in our frame, by the use of relations in Sections 3 and 4.

$$
\left\langle E_{1}(p)\left|c \alpha_{z}\right| E_{1}(p)\right\rangle=c u_{1}^{\dagger}(p) \alpha_{z} u_{1}(p)=\frac{c^{2} p}{E_{p}} .
$$

Equation (37) leads to

$$
\begin{aligned}
& \left\langle E_{1}(p)\left|v_{z}(t)^{(Z i t)}\right| E_{1}(p)\right\rangle=0 \\
& \left\langle E_{1}(p)\left|z(t)^{(Z i t)}\right| E_{1}(p)\right\rangle=R_{z}
\end{aligned}
$$

and

$$
\begin{aligned}
\left\langle E_{1}(p)\left|v_{z}(t)\right| E_{1}(p)\right\rangle & =\left\langle E_{1}(p)\left|v_{z}(t)^{(\text {unif })}\right| E_{1}(p)\right\rangle=\frac{c^{2} p}{E_{p}} \\
\left\langle E_{1}(p)|z(t)| E_{1}(p)\right\rangle & =\left\langle E_{1}(p)\left|z(t)^{(\text {unif })}\right| E_{1}(p)\right\rangle+R_{z} \\
& =\frac{c^{2} p}{E_{p}} t+z_{0}+R_{z},
\end{aligned}
$$

where $H^{-1}\left|E_{1}\right\rangle=E_{p}^{-1}\left|E_{1}\right\rangle$ is used. Arbitrary constant $z_{0}+R_{z}$ can set to zero without loss of generality. The similar results occur for $\mathrm{x}$ and y components of both $\boldsymbol{v}$ and $\boldsymbol{r}$. The contribution from uniform velocity vanish at this time because of $p_{x}=p_{y}=0$.

$$
\begin{gathered}
\left\langle v_{x}(t)^{(Z i t)}\right\rangle=\left\langle v_{y}(t)^{(Z i t)}\right\rangle=0 \\
\left\langle v_{x}(t)^{(\text {unif })}\right\rangle=\left\langle v_{y}(t)^{(\text {unif })}\right\rangle=0 \\
\left\langle x(t)^{(Z i t)}\right\rangle=R_{x}, \quad\left\langle y(t)^{(Z i t)}\right\rangle=R_{y} \\
\left\langle x(t)^{(\text {unif })}\right\rangle=x_{0}, \quad\left\langle y(t)^{(\text {unif })}\right\rangle=y_{0},
\end{gathered}
$$

where the expression of the expectation value for $\left|E_{1}\right\rangle$ is simplified. Results (38)-(46) indicate that Zitterbewegung (trembling motion) phenomenon for relativistic electron is un-observable effect in the sense that the expectation values of physical quantities always agree to classically measured one in accordance with Ehrenfest's law [20]. A question whether Zitterbewegung works or not in actual physics phenomena then arises. The answer will be shown in the next section.

\section{Spin-Magnetic Moment}

We calculate the magnetic moment based on Equation (4). As mentioned in Section 2, the velocity $v(t)$ of relativistic electron is not equal to $\boldsymbol{p} / m$ but equal to $c \boldsymbol{\alpha}(t)$. So that the expression of magnetic moment $\boldsymbol{\mu}$ must be 


$$
\boldsymbol{\mu}=\frac{e}{2} \boldsymbol{r} \times \boldsymbol{\alpha}
$$

for relativistic electron. It is our advantage that we need no external magnetic field. In what follows, we pay attention to the electron in state $\left|E_{1}\right\rangle$, and to the z-component of the magnetic moment.

$$
\begin{aligned}
\mu_{z}(t)=\frac{e}{2}\left(x(t) \alpha_{y}(t)-y(t) \alpha_{x}(t)\right)= & \frac{e}{2 c}\left[\left(x(t)^{(Z i t)}+x(t)^{(\text {unif })}\right)\left(v_{y}(t)^{(Z i t)}+v_{y}(t)^{(\text {unif })}\right)\right. \\
& \left.-\left(y(t)^{(Z i t)}+y(t)^{(u n i f)}\right)\left(v_{x}(t)^{(Z i t)}+v_{x}(t)^{(u n i f)}\right)\right] .
\end{aligned}
$$

By the use of the completeness condition

$$
\sum_{s=1}^{4} \sum_{\boldsymbol{q}}\left|E_{s}(\boldsymbol{q})\right\rangle\left\langle E_{s}(\boldsymbol{q})\right|=1
$$

we have an expression of the expectation value of $\mu_{z}$.

$$
\begin{aligned}
&\left\langle E_{1}(\boldsymbol{p})\left|\mu_{z}(t)\right| E_{1}(\boldsymbol{p})\right\rangle \\
&=\sum_{s=1}^{4} \sum_{\boldsymbol{q}} \frac{e}{2 c} \times\left[\left\langle E_{1}(\boldsymbol{p})\left|x(t)^{(Z i t)}\right| E_{s}(\boldsymbol{q})\right\rangle\left\langle E_{s}(\boldsymbol{q})\left|v_{y}(t)^{(Z i t)}\right| E_{1}(\boldsymbol{p})\right\rangle\right. \\
&+\left\langle E_{1}(\boldsymbol{p})\left|x(t)^{(Z i t)}\right| E_{s}(\boldsymbol{q})\right\rangle\left\langle E_{s}(\boldsymbol{q})\left|v_{y}(t)^{(u n i f)}\right| E_{1}(\boldsymbol{p})\right\rangle \\
&+\left\langle E_{1}(\boldsymbol{p})\left|x(t)^{(u n i f)}\right| E_{s}(\boldsymbol{q})\right\rangle\left\langle E_{s}(\boldsymbol{q})\left|v_{y}(t)^{(\text {Zit })}\right| E_{1}(\boldsymbol{p})\right\rangle \\
&+\left\langle E_{1}(\boldsymbol{p})\left|x(t)^{(u n i f)}\right| E_{s}(\boldsymbol{q})\right\rangle\left\langle E_{s}(\boldsymbol{q})\left|v_{y}(t)^{(\text {unif })}\right| E_{1}(\boldsymbol{p})\right\rangle \\
&-\left\langle E_{1}(\boldsymbol{p})\left|y(t)^{(\text {Zit })}\right| E_{s}(\boldsymbol{q})\right\rangle\left\langle E_{s}(\boldsymbol{q})\left|v_{x}(t)^{(Z i t)}\right| E_{1}(\boldsymbol{p})\right\rangle \\
&-\left\langle E_{1}(\boldsymbol{p})\left|y(t)^{(\text {Zit })}\right| E_{s}(\boldsymbol{q})\right\rangle\left\langle E_{s}(\boldsymbol{q})\left|v_{x}(t)^{(u n i f)}\right| E_{1}(\boldsymbol{p})\right\rangle \\
&-\left\langle E_{1}(\boldsymbol{p})\left|y(t)^{(\text {unif })}\right| E_{s}(\boldsymbol{q})\right\rangle\left\langle E_{s}(\boldsymbol{q})\left|v_{x}(t)^{(\text {Zit })}\right| E_{1}(\boldsymbol{p})\right\rangle \\
&\left.-\left\langle E_{1}(\boldsymbol{p})\left|y(t)^{(\text {unif })}\right| E_{s}(\boldsymbol{q})\right\rangle\left\langle E_{s}(\boldsymbol{q})\left|v_{x}(t)^{(\text {unif })}\right| E_{1}(\boldsymbol{p})\right\rangle\right]
\end{aligned}
$$

Each matrix elements of uniform part are calculated as follows:

$$
\begin{gathered}
\left\langle E_{s}(\boldsymbol{q})\left|v_{x}(t)^{(\text {unif })}\right| E_{1}(\boldsymbol{p})\right\rangle=\left\langle E_{s}(\boldsymbol{q})\left|v_{y}(t)^{(\text {unif })}\right| E_{1}(\boldsymbol{p})\right\rangle=0 \\
\left\langle E_{1}(\boldsymbol{p})\left|x(t)^{(u n i f)}\right| E_{s}(\boldsymbol{q})\right\rangle=x_{0} \delta_{1 s} \delta_{\boldsymbol{p q}} \\
\left\langle E_{1}(\boldsymbol{p})\left|y(t)^{(\text {unif })}\right| E_{s}(\boldsymbol{q})\right\rangle=y_{0} \delta_{1 s} \delta_{\boldsymbol{p q}}
\end{gathered}
$$

We have also Zitterbewegung part of the velocity.

$$
\left\langle E_{s}(\boldsymbol{q})\left|v_{x}(t)^{(Z i t)}\right| E_{1}(\boldsymbol{p})\right\rangle=u_{s}^{\dagger}(\boldsymbol{q}) c \alpha_{x} u_{1}(\boldsymbol{p}) \exp \left(\frac{2 E_{p} t}{i \hbar}\right) \delta_{q p}=c \exp \left(\frac{2 E_{p} t}{i \hbar}\right) \delta_{4 s} \delta_{q p}
$$

as well as

$$
\left\langle E_{s}(\boldsymbol{q})\left|v_{y}(t)^{(Z i t)}\right| E_{1}(\boldsymbol{p})\right\rangle=i c \exp \left(\frac{2 E_{p} t}{i \hbar}\right) \delta_{4 s} \delta_{q p} .
$$

Substitution of Equations (51)-(55) into Equation (50) gives

$$
\begin{aligned}
\left\langle E_{1}(\boldsymbol{p})\left|\mu_{z}(t)\right| E_{1}(\boldsymbol{p})\right\rangle= & \sum_{q} \frac{e}{2 c}\left(\left\langle E_{1}(\boldsymbol{p})\left|x(t)^{(Z i t)}\right| E_{4}(\boldsymbol{q})\right\rangle\left\langle E_{4}(\boldsymbol{q})\left|v_{y}(t)^{(Z i t)}\right| E_{1}(\boldsymbol{p})\right\rangle\right. \\
& \left.-\left\langle E_{1}(\boldsymbol{p})\left|y(t)^{(Z i t)}\right| E_{4}(\boldsymbol{q})\right\rangle\left\langle E_{4}(\boldsymbol{q})\left|v_{x}(t)^{(Z i t)}\right| E_{1}(\boldsymbol{p})\right\rangle\right) .
\end{aligned}
$$


We find here that $\left\langle\mu_{z}\right\rangle$ is made only by Zitterbewegung parts. We can easily obtain each element:

$$
\begin{gathered}
\left\langle E_{1}(\boldsymbol{p})\left|x(t)^{(Z i t)}\right| E_{4}(\boldsymbol{q})\right\rangle=i\left\langle E_{1}(\boldsymbol{p})\left|y(t)^{(Z i t)}\right| E_{4}(\boldsymbol{q})\right\rangle=-\frac{i \hbar c}{2 E_{p}} \exp \left(-\frac{2 E_{p} t}{i \hbar}\right) \delta_{p q} . \\
\left\langle E_{4}(\boldsymbol{q})\left|v_{y}(t)^{(Z i t)}\right| E_{1}(\boldsymbol{p})\right\rangle=i\left\langle E_{4}(\boldsymbol{q})\left|v_{x}(t)^{(Z i t)}\right| E_{1}(\boldsymbol{p})\right\rangle=i c \exp \left(\frac{2 E_{p} t}{i \hbar}\right) \delta_{q p} .
\end{gathered}
$$

We then finally obtain

$$
\left\langle E_{1}(\boldsymbol{p})\left|\mu_{z}(t)\right| E_{1}(\boldsymbol{p})\right\rangle=\frac{e \hbar c}{2 E_{p}}
$$

which is the same result as in the previous work [2].

It is necessary to recall that there is not any z-component of magnetic moment arising from orbital motion of electron because of $\boldsymbol{p}=(0,0, p)$ and $L_{z}=0$ in our frame. Nevertheless, magnetic moment of Equation (59) has actually appeared. Therefore, we may conclude that $\mu_{z}$ of Equation (59) and the spin-magnetic moment of the electron must be identified. When the momentum of the electron is small and $E_{p} \cong m c^{2}$, it becomes

$$
\left\langle E_{1}(\boldsymbol{p})\left|\mu_{z}(t)\right| E_{1}(\boldsymbol{p})\right\rangle=\frac{e \hbar}{2 m c},
$$

indicating correct $g$-factor because of $\left\langle E_{1}\left|S_{z}\right| E_{1}\right\rangle=\hbar / 2$ for Dirac electron in our frame; that is

$$
\left\langle E_{1}(p)\left|\mu_{z}(t)\right| E_{1}(p)\right\rangle=g \frac{e}{2 m c}\left\langle E_{1}(p)\left|S_{z}\right| E_{1}(p)\right\rangle \quad(g=2),
$$

where $S$ is the $4 \times 4$ spin matrix operator.

\section{Concluding Remarks}

As seen in Sections 5, the expectation values of all Zitterbewegung parts give zero or constant, both in velocity and coordinates. This means that we can not directly measure the effect of Zitterbewegung. However, they still survive behind some kind of physical quantities. The magnetic moment is an example. Although Zitter-bewegung relating to the velocity or coordinates is un-observable in the sense of the expectation value which corresponds to classical behavior, we have shown that it exists and works through the magnetic moment. A crucial point is non-diagonal matrix elements: $\left\langle E_{4}\left|e v_{i}(t)^{(Z i t)}\right| E_{1}\right\rangle(i=x, y)$. The physical meaning of these matrix elements is inferred as follows:

The initial state $\left|E_{1}\right\rangle$ of electron with positive energy and up-spin undergoes transition into the state $\left|E_{4}\right\rangle$ with opposite signs of energy and spin (See Equations (29) and (30)), by operating $e v_{i}(t)^{(Z i t)}$. This matrix element is a kind of "transition current" because it exactly corresponds to the electric current $\boldsymbol{J}$ in the definition of magnetic moment [2] which is written in another form [18],

$$
\boldsymbol{\mu}=\frac{1}{2 c} \int \boldsymbol{r} \times \boldsymbol{J d}^{3} \boldsymbol{r}
$$

In quantum theory, the conservation law of energy may break by $\Delta E$ in time $\Delta t$, where

$$
\Delta E \Delta t \cong \hbar \text {. }
$$

The electron which has undergone transition into $\left|E_{4}\right\rangle$ must immediately return to $\left|E_{1}\right\rangle$ within time $\Delta t$. The period of transition cycle is about

$$
\omega \simeq 1 / \Delta t \cong \Delta E / \hbar \cong 2 m c^{2} / \hbar,
$$

where $\Delta E \cong 2 m c^{2}$ means the energy gap of Dirac Sea in vacuum. This $\omega$ agrees with the frequency of Zitterbewegung (See Equations (57) and (58)).

In the classical electrodynamics, the magnetic moment is caused by periodic orbital motion of a charged particles which is equivalent to an electric current. In the relativistic quantum theory, it seems that it is possible to cause the magnetic moment also by periodic transition from the positive energy state to the negative energy state (Figure 1). It then seems that the former is the magnetic moment which corresponds to Equation (5) with 


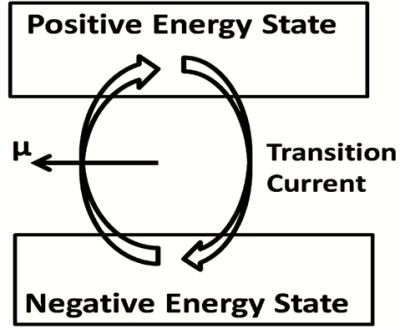

Figure 1. Spin-magnetic moment caused by transition current.

$g=1$, and the latter is the spin-magnetic moment with $g=2$. In other word, the spin-magnetic moment may be caused not by usual electric current but by some new current which yield when the electron undergoes transition between two states of positive and negative energies. It should be noted that even an electron at rest (i.e. $\boldsymbol{p}=0$ and $\boldsymbol{L}=0$ ) in space is able to yield this new current.

\section{Another Remarks}

Such a situation as described above occurs in some kind of solid state. In the two band model of Cohen and Blount [21], Wolff [22] indicated that the Hamiltonian takes the Dirac form after a suitable transformation, and that the resulting equations are essentially identical to those of the Dirac theory. This fact means that we could apply the method developed here to the spin-magnetic moment of electron in solid state [23]. Zawadzki [11] indeed pointed out that the energy of the electron in narrow-gap semiconductor was given as

$$
E_{p}=\sqrt{p^{2} u^{2}+m^{\star 2} u^{4}}
$$

( $m^{\star}$ : effective mass), where $u$ is maximum value of the group velocity $v_{g}$ of electron in band:

$$
u \equiv \lim _{p \rightarrow \infty} v_{g}
$$

The Hamiltonian which corresponds to Equation (65) has the form

$$
H=\alpha_{3} u p_{0}+\beta m^{\star} u^{2}
$$

in the frame

$$
\boldsymbol{p}=\left(0,0, p_{0}\right)
$$

where $\boldsymbol{\alpha}=\left(\alpha_{1}, \alpha_{2}, \alpha_{3}\right)$ and $\beta$ are the Dirac matrices. The above Hamiltonian agrees completely to the Dirac Hamiltonian in Equation (7) if we replace $u$ by $c$, and $m^{\star}$ by $m$. Obeying the Heisenberg equation of motion, it is then predicted that $H$ of the equal form reproduces the same results obtained here with the replacements $c \rightarrow u$ and $m \rightarrow m^{\star}$. They will appear as a change of $g$-factor of the electron in semiconductor from new equation corresponding to Equation (59).

\section{References}

[1] Žutić, I., Fabian, J. and Sarma, S.D. (2004) Reviews of Modern Physics, 76, 323-410. http://dx.doi.org/10.1103/RevModPhys.76.323

[2] Sasabe, S. and Tsuchiya, K. (2008) Physics Letters A, 372, 381-386. http://dx.doi.org/10.1016/j.physleta.2007.07.078

[3] Schrödinger, E. (1930) Sitzungsber. Preuss. Akad. Wiss. Phys. Math. Kl., 24, 418-428.

[4] Barut, A.O. and Bracken, A.J. (1981) Physical Review D, 23, 2454-2463. http://dx.doi.org/10.1103/PhysRevD.23.2454

[5] Sasabe, S. (2000) Electrical Engineering in Japan, 132, 1-6. http://dx.doi.org/10.1002/(SICI)1520-6416(20000715)132:1<1::AID-EEJ1>3.0.CO;2-O

[6] Castro, A.H., et al. (2009) Reviews of Modern Physics, 81, 109-162. http://dx.doi.org/10.1103/RevModPhys.81.109

[7] Zawadzki, W. and Rusin, T.M. (2011) Journal of Physics: Condensed Matter, 23, 143201. http://dx.doi.org/10.1088/0953-8984/23/14/143201

[8] Cannata, F., Ferrari, L. and Russo, G. (1990) Solid State Communications, 74, 309-312. 
http://dx.doi.org/10.1016/0038-1098(90)90192-E

[9] Ferrari, L. and Russo, G. (1990) Physical Review B, 42, 7454-7461. http://dx.doi.org/10.1103/PhysRevB.42.7454

[10] Rusin, T.M. and Zawadzki, W. (2007) Physical Review B, 76, 195439.

[11] Zawadzki, W. (2005) Physical Review B, 72, 085217. http://dx.doi.org/10.1103/PhysRevB.72.085217

[12] Katsnelson, M. (2006) The European Physical Journal B, 51, 157-160. http://dx.doi.org/10.1140/epjb/e2006-00203-1

[13] Zawadzki, W. (2006) Physical Review B, 74, 205439. http://dx.doi.org/10.1103/PhysRevB.74.205439

[14] Lurié, D. and Cremer, S. (1970) Physica, 50, 224-240. http://dx.doi.org/10.1016/0031-8914(70)90004-2

[15] Dirac, P.A.M. (1958) The Principle of Quantum Mechanics. Oxford University Press, Section 70.

[16] Schiff, L.I. (1968) Quantum Mechanics. McGraw-Hill, Section 43.

[17] Jackson, J.D. (1975) Classical Electrodynamics. John Wiley \& Sons, New York, Section 5.6.

[18] Landau, L.D. and Lifschitz, E.M. (1975) The Classical Theory of Fields. Pergamon Press, Section 44.

[19] Dirac, P.A.M. (1958) The Principle of Quantum Mechanics. Oxford University Press, Section 67-69.

[20] Schiff, L.I. (1968) Quantum Mechanics. McGraw-Hill, Section 7.

[21] Cohen, M.H. and Blount, E.I. (1960) Philosophical Magazine, 5, 115-126. http://dx.doi.org/10.1080/14786436008243294

[22] Wolff, F.A. (1964) Journal of Physics and Chemistry of Solids, 25, 1057-1068. http://dx.doi.org/10.1016/0022-3697(64)90128-3

[23] Sasabe, S. and Tsuchiya, K. (2008) T. IEE Japan A, 128, 313-314. 